\title{
Sustainability Mindset: a challenge for educational professions?
}

\author{
Sustainability Mindset: una sfida per le professioni educative?
}

\author{
Giovanna Del Gobboa \\ a Università degli Studi di Firenze, giovanna.delgobbo@unifi.it
}

Il tema della sostenibilità intesa nelle sue diverse componenti - ambientali, sociali, economiche e culturali (UCLG, 2010; UN, 1995) - si sta sempre più affermando come asset trasversale: è la parola d'ordine dell'Agenda 2030 delle Nazioni Unite, che, con i 17 obiettivi, intende indicare la direzione di cambiamenti strutturali a livello globale, spingendo sull'innovazione e sulla ridefinizione delle priorità delle società e dei modelli economici, sulle abitudini di vita (EEA \& Eionet, 2017; EEA, 2015; 2019; Singh, 2012; Varey, 2012).

Nel 1987 la World Commission on Environment and Development (WCED) introduceva il costrutto di sviluppo sostenibile in termini di sviluppo che soddisfa i bisogni del presente senza compromettere la capacità delle generazioni future di soddisfare i propri bisogni (Rapporto Brundtland - UN, 1987): uno sviluppo con al centro l'attenzione all'ambiente, in una prospettiva economica e sociale. Nel 1991 il documento Caring for the Earth, A strategy for Sustainable Living, elaborato da IUNC (The world conservation union), UNEP (United Nation Environment Programme) e WWF (World Wide Fund for Nature), utilizzava il costrutto di sviluppo sostenibile per significare "improving the quality of human life while living within the carrying capacity of supporting ecosystem" (IUNC, UNEP, \& WWF, 1991, p. 10). Il modello di sostenibilità si è andato definendo attraverso obiettivi di miglioramento dell'ecosistema e del benessere umano.

La sostenibilità stessa rappresenta, dunque, un obiettivo che per essere raggiunto richiede processi e percorsi di sviluppo sostenibile. Gli SDGs (Sustainable Development Goals) forniscono un quadro di riferimento per affrontare problemi globali come povertà, disoccupazione, disuguaglianze e disparità legate al genere, alla distribuzione iniqua di ricchezza e potere, ai rischi ambientali. I 17 Obiettivi e i 169 traguardi rappresentano una strategia ambiziosa per affrontare problemi urgenti e ineludibili.

In questo quadro appare evidente la necessità di un cambiamento culturale, di un orientamento complessivo e pervasivo alla sostenibilità. Perseguire uno sviluppo sostenibile richiede quello che è stato definito come sustainability mindset (Hermes \& Rimanoczy, 2018; Kassel, Rimanoczy, \& Mitchell, 2016; Rimanoczy, 2021), un cambiamento di mentalità che comprende valori, atteggiamenti e comportamenti, un approccio sistemico che va oltre la conoscenza tecnica e si concentra invece su una più ampia comprensione dell'ecosistema e della sostenibilità (Kassel \& Rimanoczy, 2018).

Anche in ambito educativo, ormai da alcuni anni, il dibattito si è spostato da una educazione ambientale (con focus prevalente sulla cura dell'ambiente e della biodiversità) all'educazione allo sviluppo sostenibile, con attenzione ai temi dell'economia, della salute, della povertà e dell'inclusione sociale. La maggiore caratterizzazione dell'educazione ambientale in termini di educazione allo sviluppo ha portato a riconoscere all'educazione una funzione chiave (Marcinkowski, 2009). La capacità di interpretare e agire in coerenza con i principi della sostenibilità viene ad essere 
considerata sempre più necessaria e qualificante in tutti i contesti. Le competenze green richieste dal mercato del lavoro si presentano non tanto come tecniche e specifiche, quanto come un orientamento personale e una sensibilità e propensione culturale: si fondano sulla consapevolezza ambientale e sull'orientamento allo sviluppo sostenibile nei comportamenti e negli stili di vita e in quanto tali risultano fortemente derivanti da una pluralità di opportunità educative (educazione ai consumi, educazione alimentare, educazione alla gestione dei rifiuti e al risparmio energetico, etc.). Lo sviluppo di queste competenze richiede un approccio lifelong lifewide oriented dei processi educativi che non possono esaurirsi nei contesti di istruzione, ma richiedono di essere sostenuti e rafforzati in un pluralità/continuità di esperienze e di ambienti di apprendimento. L'ambito educativo no-teaching (educazione non formale e informale) prevalentemente legato al Terzo Settore, svolge di fatto una funzione importante nel campo dell'educazione alla sostenibilità ambientale (come previsto anche dalla L. n. 106/2016 per la riforma del Terzo settore, dell'impresa sociale e per la disciplina del servizio civile universale) anche in collaborazione con il sistema dell'istruzione per la realizzazione delle attività e il raggiungimento dei risultati di apprendimento previsti dai programmi e documenti ministeriali (dal Programma INFormazione Educazione Ambientale, INFEA, alle Linee Guida per l'educazione ambientale del 2009 e del 2015, sino alle recentissime Linee guida per l'insegnamento dell'educazione civica). È sempre l'ambito professionale del no-teaching che svolge un ruolo fondamentale di sensibilizzazione, educazione e sviluppo di consapevolezza sulle tematiche della sostenibilità ambientale, sia nel Terzo Settore (basti pensare alle azioni educative sviluppate da Fondazioni, Organizzazioni non Governative, ONLUS o da parte dei molteplici soggetti della società civile organizzata che si occupano di temi ambientali) sia all'interno di soggetti pubblici (come l'Istituto Superiore per la Protezione la Ricerca Ambientale, ISPRA, le Agenzie regionali per la Protezione ambientale, ARPA, o i Centri e i Laboratori di educazione ambientale collegati alle istituzioni locali).

A fronte della rilevanza che, per rispondere alle esigenze di sviluppo dell'attitudine green da parte di lavoratori e cittadini, viene riconosciuta indirettamente alla famiglia professionale dell'educatore in quanto professionista dell'educazione non formale, i servizi e le azioni educative e i professionisti, che già operano per queste finalità, sono di difficile identificazione e non solo a livello nazionale

Le professioni educative e formative (teaching e no-teaching), benché non rientrino propriamente nei cosiddetti green jobs, sono così chiamate in causa da un duplice punto di vista, di cui il primo appare funzionale al secondo:

1. ai professionisti dell'educazione e della formazione sono richieste specifiche competenze;

2. ai professionisti dell'istruzione, dell'educazione e della formazione è attribuito un ruolo importante per lo sviluppo delle competenze necessarie ad una transizione eco-sostenibile.

In questo quadro il numero della rivista ha raccolto contributi di riflessione ed esperienze educative che consentono di evidenziare la significatività di un'azione educativa critica e responsabile. Un'azione a sostegno di un futuro sostenibile sul piano ambientale, economico, culturale, sociale e umano, come sottolinea Simona Sandrini nel suo contributo, evidenziando l'integrazione inscindibile tra transizione verde e umana, che le professioni pedagogiche sono chiamate a garantire come orizzonte di senso per aiutare le giovani generazioni a ricomporre un intero umano, evitando il rischio di appiattire la sostenibilità su dispositivi puramente funzionali. Da qui la proposta di predisporre 
ambienti formativi in cui $\mathrm{i}$ giovani sperimentino la cultura dell'incontro e della prossimità.

Patrizia Galeri sviluppa invece la riflessione sulla sostenibilità in riferimento alla cultura del territorio, evidenziando la necessità di progetti turistici sostenibili per rispondere alle esigenze di sviluppo equo e solidale. Il turismo sostenibile è considerato per la profonda sintonia con i temi e le prospettive dello sviluppo umano e inquadrato come straordinario driver per potenziare il placement: la prospettiva del turismo sostenibile e la formazione di professionisti con adeguate capacità, è connessa con un'interpretazione delle attuali curvature e risultanze euristiche di una green economy capace di generare nuovi posti di lavoro e attività creativo-innovative.

$\mathrm{Su}$ una concezione ecosostenibile della salute, del benessere e dello stile di vita sia a livello individuale che collettivo, si sofferma Vincenzo Armendolagine, con l'obiettivo di esplicitare i raccordi che esistono fra salute, benessere, stile di vita e sviluppo del substinability mindset. Sono analizzati gli elementi costitutivi del concetto di benessere, di salute mentale e di disturbo mentale, successivamente sono illustrati i costrutti di salute, patologia e determinanti di salute e le implicazioni che essi hanno nella genesi dello stile di vita, concludendo con una proposta di strategie possibili per cambiare gli stili vita nell'ottica della sostenibilità.

$\mathrm{Su}$ strategie didattiche per educare allo sviluppo sostenibile in ambienti di alta formazione si sofferma invece il contributo di Del Gobbo, De Maria e Pampaloni presentando un'esperienza di insegnamento all'interno di un corso di laurea magistrale di area pedagogica dell'Università di Firenze. L'articolo evidenzia l'importanza di approcci pedagogici e didattici student centred e learning outcomes oriented funzionali allo sviluppo di competenze legate al tema dello sviluppo sostenibile e volti alla formazione di professionisti competenti per l'accompagnamento e la gestione di processi di transizione ecologica e sostenibile.

Il numero offre anche alcuni articoli con focus differenziati, non correlati al tema dell'educazione allo sviluppo sostenibile.

Sulla competenza etica dell'insegnante si sofferma il contributo di Paolo Calidoni, Filippo Dettori, Giusy Manca e Luisa Pandolfi. È evidenziata la rilevanza e la trasversalità di questa competenza per la gestione delle dinamiche della classe e dei rapporti con le famiglie e con i colleghi e in particolare per le attività di sostegno didattico agli alunni con disabilità. Il contributo presenta una ricerca esplorativa realizzata presso l'Università di Sassari, nell'ambito di un PRIN sull'educazione morale nella scuola secondaria di primo grado, finalizzata a definire adeguate strategie formative per lo sviluppo di competenze etiche, riflessive ed argomentative.

Il contributo di Laura Menichetti e Silvia Micheletta analizza la letteratura scientifica dal 2000 al 2021 per determinare in quali contesti formativi siano utilizzati i makerspace e con quali finalità, per bambini e ragazzi di età prescolare e scolare. I makerspace sono comunità reali centrate sul fare e costruire, luoghi dove poter dare forma ai propri progetti. La scoping review mostra che in letteratura sono documentate molte esperienze, ma esistono pochi studi sperimentali. Gli esempi raccolti, di stampo costruzionista, mostrano la validità del dispositivo per lo sviluppo di competenze di problem solving e creatività, per una più consapevole applicazione nelle discipline tecnico-scientificheartistiche, per la realizzazione di contesti inclusivi soprattutto in situazioni di disagio sociale. 
Silvia Dell'Anna e Dario Ianes affrontano invece il tema della dispersione scolastica: una questione di grande rilevanza nel nostro Paese, sia per le sue conseguenze individuali sia per le ricadute sociali ed economiche. L'articolo riflette sul fenomeno dell'abbandono scolastico precoce e sulle opzioni di intervento, con particolare riferimento al periodo di emergenza sanitaria. Nel quadro di una esperienza realizzata nel 2020, il contributo prende in esame fattori protettivi e rischio, bisogni emergenti, contenuti e strategie didattiche.

Davide Forgione si sofferma sul tema delle competenze di progettazione di coloro che operano in ambito educativo e formativo, e sulla necessità di saper individuare metodi e pratiche pedagogiche vicine al contesto culturale degli individui. Prendendo come riferimento i beneficiari pre-adolescenti e adolescenti di un centro educativo in un'area urbana è formulata un'ipotesi di intervento di educativa di strada mediate la Hip-Hop Based Education, un insieme di pratiche che incorporano gli elementi creativi della cultura hip-hop nell'azione educativa.

Le revisioni sistematiche sono l'oggetto del contributo di Marta Pellegrini e Francesco Marsili e in particolare l'utilizzo di software specifici, attualmente poco diffusi nella ricerca educativa italiana. Il contributo presenta quattro software utilizzati nella ricerca educativa internazionale e valuta le funzionalità generali e l'usabilità di caratteristiche specifiche per condurre le fasi di screening e di selezione degli studi.

La parte monografica del numero si completa con la presentazione di tre contributi di riflessione collegate ad esperienze.

Il contributo di Sara Bornatici e Orietta Vacchelli è centrato sulla recente normativa, la L. n. 92/2019, che ha introdotto dall'anno scolastico 2020-2021 l'insegnamento trasversale dell'educazione civica nelle scuole di ogni ordine e grado. Il contributo presenta alcuni dati emersi nell'ambito della ricerca CIVES (CIttadini VErso la Sostenibilità), scaturiti da un'esperienza di formazione degli insegnanti che ha visto la cultura della sostenibilità e la cittadinanza solidale come due possibili chiavi di lettura della proposta legislativa.

Caterina Braga presenta il progetto CLIC-PLAN (CLIma in Cambiamento. Piano Locale di AdattameNto) dell'Università Cattolica, finalizzato a sostenere alcuni comuni lacustri in territorio subalpino con forte vocazione turistica attraverso azioni di educazione alla partecipazione per promuove il senso di appartenenza e rendere i cittadini attori responsabili dei processi decisionali di gestione del proprio territorio. Obiettivo è rendere i singoli individui e le comunità responsabili della vita sociale a tutti i livelli (economicopolitico, istituzionale, amministrativo, produttivo, culturale) e consapevoli dell'impatto delle proprie azioni sull'ambiente.

Teresa Giovannazzi nel suo contributo riprende il filo rosso dell'individuare modi più equilibrati e autentici per abitare la Terra e evidenzia come questo implichi la promozione di azioni educative efficaci, tra responsabilità e valori condivisi, fin dalle professionalità educative 0-6.

\section{Riferimenti bibliografici}

EEA, Eionet. European Environment Agency, European Environment Information and Observation Network (2017). Mapping Europe's environmental future: understanding the impacts of global megatrends at the national level. Luxembourg: Publications Office of the European Union. 
EEA. European Environment Agency (2015). European environment - state and outlook 2015: Assessment of global megatrends. Copenhagen: European Environment Agency.

Hermes, J., \& Rimanoczya, I. (2018). Deep learning for a sustainability mindset. The International Journal of Management Education, 16(3), 460-467.

IUNC, UNEP, WWF. International Union for Conservation of Nature and Natural Resources, United Nations Environment Programme, World Wildlife Fund (1991). Caring for the Earth. A Strategy for a sustainable Living. Gland, Switzerland.

Kassel, K., \& Rimanoczy, I. (Eds.). (2018). Developing a sustainability mindset in management education. New York, NY: Routledge.

Kassel K., Rimanoczy I., Mitchell S. (2016), The Sustainable Mindset: Connecting Being, Thinking, and Doing in Management Education, Academy of Management Annual Meeting Proceedings 2016(1):16659.

Legge 20 agosto 2019, n. 92. Introduzione dell'insegnamento scolastico dell'educazione civica.

Legge 6 giugno 2016, n. 106. Delega al Governo per la riforma del Terzo settore, dell'impresa sociale e per la disciplina del servizio civile universale.

Marcinkowski, T. (2009). Contemporary challenges and opportunities in environmental education: Where are we headed and what deserves our attention? The Journal of Environmental Education, 41(1), 34-54.

MIUR, MITE. Ministero dell'Istruzione dell'Università e della Ricerca. Ministero dell'Ambiente e della Tutela del Territorio e del Mare (2009). Linee guida per l'educazione ambientale e allo sviluppo sostenibile. https://www.mite.gov.it/sites/default/files/archivio/notizie/Linee_guida_Scuolax Ambiente_e Legalitx_aggiornato.pdf (ver. 15.07.2021).

MIUR, MITE. Ministero dell'Istruzione dell'Università e della Ricerca. Ministero dell'Ambiente e della Tutela del Territorio e del Mare (2015). Linee guida per l'educazione ambientale e allo sviluppo sostenibile. https://www.mite.gov.it/sites/default/files/archivio/allegati/LINEE_GUIDA.pdf (ver. 15.07.2021).

Rimanoczy, I. (2021). The sustainability mindset principles. A guide to developing a mindset for a better world. New York, NY: Routledge.

Singh, S. (2012). New mega trends. Implications for our future lives. London: Palgrave Macmillan.

UN. United Nations (1987). Report of the World Summit for Social Development. Our common future. United Nations.

UN. United Nations (1995). Report of the World Summit for Social Development $A / C O N F .166 / 9$. United Nations.

UCLG. United Cities and Local Governments (2010). The world summit of local and regional leaders. Third UCLG World Congress, Città del Messico, Messico.

Varey, R. (2012). The marketing future beyond the limits of growth. Journal of Macromarketing, 32(4), 424-433. 